\title{
EXPERIMENTAL SIMULATION OF HEAT AND STRESS FORMATION FOR SURFACE GRINDING
}

\author{
BILEK, O. \& LUKOVICS, I.
}

Abstract: The article describes an application of a FEM simulation for grinding process. The further experimental computer simulation solves the influence of abrasive grain wear on the output parameters such as heat generation, chip temperature and stress formation; a grinding grain was substituted by an approximate sharp and worn model.

Key words: FEM, Grinding, Simulation, Heat, Grinding Grain
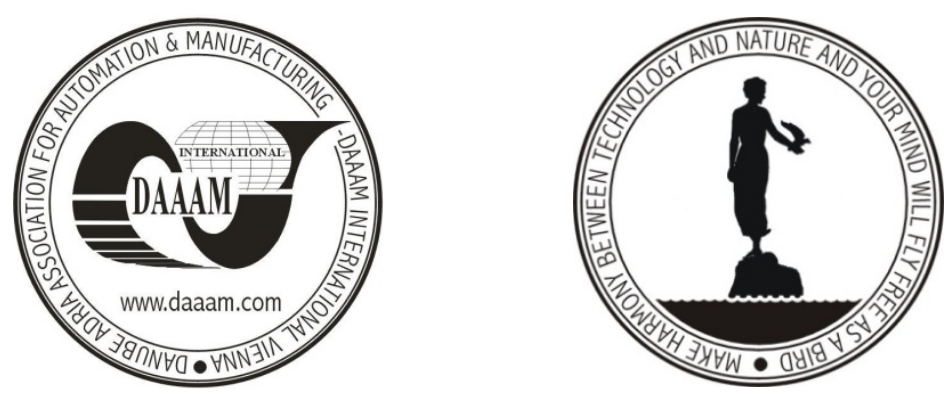

Authors' data: Prof. Bilek, O[ndrej]; Lukovics, I[mrich]; Tomas Bata University in Zlin, Nam.TGM 5555, 76001, Zlin, CZ, bilek@ft.utb.cz, lukovics@ft.utb.cz

This Publication has to be referred as: Bilek, O[ndrej] \& Lukovics, I[mrich] (2008). Experimental Simulation of Heat and Stress Formation for Surface Grinding, Chapter 04 in DAAAM International Scientific Book 2008, pp. 035-042, B. Katalinic (Ed.), Published by DAAAM International, ISBN 978-3-901509-66-7, ISSN 17269687, Vienna, Austria

DOI: $10.2507 /$ daaam.scibook.2008.04 